\title{
Avaliação da anticoagulação natural em pacientes com diabetes mellitus tipo 2
}

\section{Natural anticoagulation evaluation in type 2 diabetes}

Anna Letícia Soares'; Marcelo Carvalho Lasmar'; Micheline Lopes Garcia'; Bethânia Alves Novelli²; Geralda de Fátima Guerra Lages'; Luci Maria Sant'Ana Dusse'; Lauro Mello Vieira'; Ana Paula Salles Moura Fernandes'; Marinez de Oliveira Sousa'; Maria das Graças Carvalho

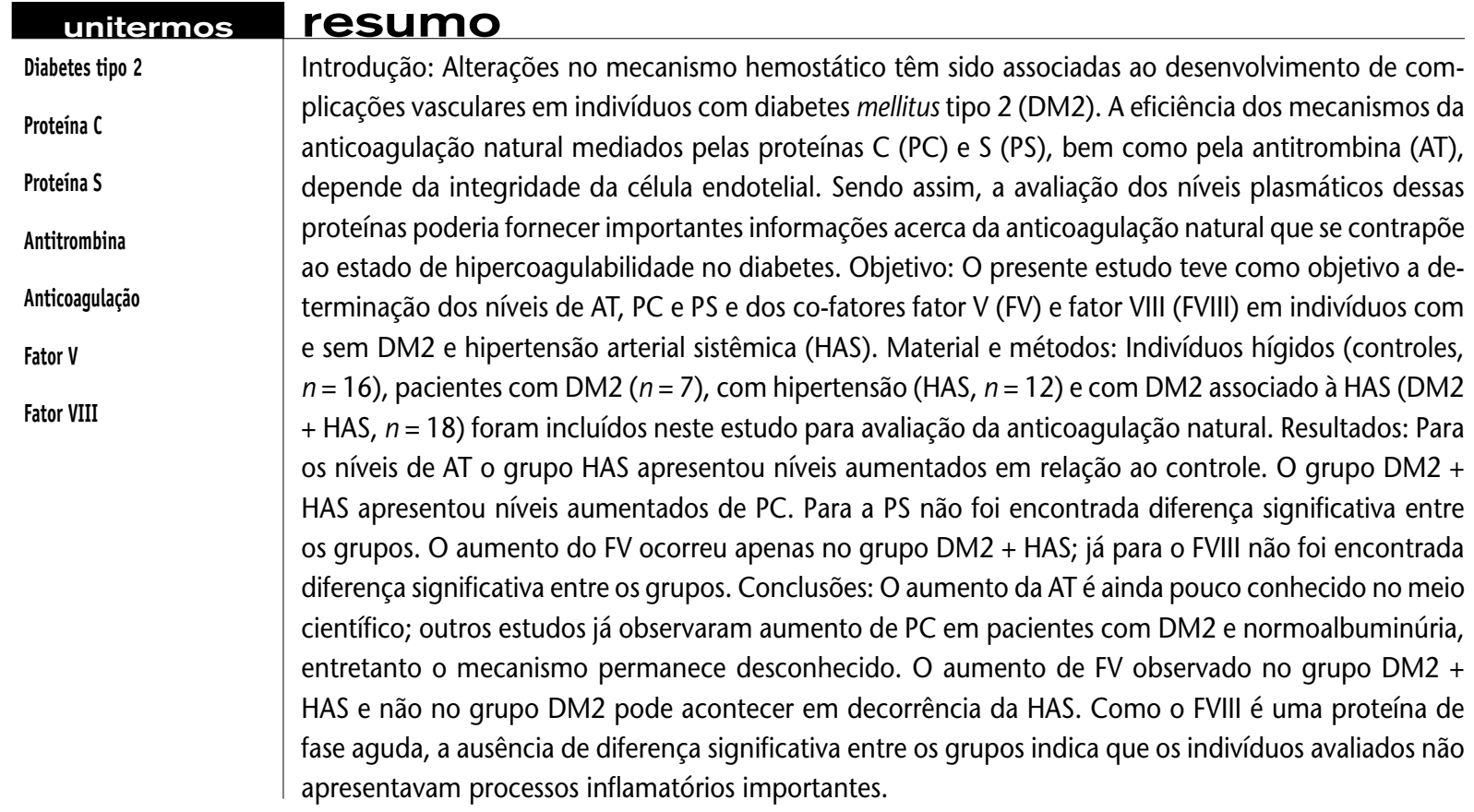

abstract

Background: Hemostatic abnormalities have been associated with vascular complications in patients with type 2 diabetes (DM2). Efficiency of the natural anticoagulation mechanism mediated by proteins $C(P C), S$ (PS) and antithrombin (AT) depends on intact endothelial cells, so the evaluation of these proteins may contribute to a better understanding of the natural anticoagulation status, considering that they inhibit the state of hypercoagulability. Objectives: The aim of this study was to measure AT, PC and PS levels and the cofactors, factors $V$ (FV) and VIII (FVIII), in subjects with and without DM2 and hypertension (HAS). Material and method: 16 healthy subjects (controls), seven patients with DM2, 12 with hypertension (HAS) and 18 with DM2 associated to HAS (DM2 + HAS) were included in this study for natural anticoagulation evaluation. Results: HAS group showed increased levels of AT compared with controls. DM2 + HAS group showed increased levels of PC. For PS and FVIII, no difference was observed among groups. Nevertheless, the increase of FV was observed in DM2 + HAS group, while DM2 group did not show increase. Conclusion: AT increase is not yet well known for all conditions. Previous studies have already described PC increase in normoalbuminuric patients with DM2, however its cause is also unknown. FV increase observed in DM2 + HAS group suggests that HAS should contribute to this increase. As FVIII is an acute phase protein the data for FVIII may indicate that assessed subjects had no important inflammatory condition. key words

Type 2 diabetes

Protein C

Protein S

Antithrombin

Anticoagulation

Factor $V$

Factor VIII 


\section{Introdução}

O diabetes mellitus (DM) é um importante problema de saúde pública, considerando-se a alta freqüência e o fato de que as suas complicações comprometem a produtividade, a qualidade de vida e a sobrevida dos indivíduos, além de envolver altos custos no tratamento da doença primária e das complicações ${ }^{(19,28)}$. O DM tipo 2 é a forma mais freqüente e corresponde a cerca de $90 \%$ dos casos de DM no mundo(27).

Dados da Organização Mundial de Saúde (OMS) referentes ao ano de 2000 mostram que aproximadamente 150 milhões de pessoas no mundo são diabéticas, e estima-se que esse número deva dobrar até o ano de 2025. Esse aumento no número de casos de diabetes deverá ocorrer principalmente em países que estão em desenvolvimento e será decorrente de aumento da população, aumento da expectativa de vida, padrão alimentar pouco saudável, obesidade e estilo de vida sedentário(8, 27).

Dados de 2000 indicam que $80 \%$ dos pacientes com DM morrem por trombose e $75 \%$ dessas mortes resultam de eventos cardiovasculares. Anormalidades no sistema hemostático, congênitas ou adquiridas, são fatores de risco importantes para os indivíduos diabéticos ${ }^{(2)}$.

O DM tipo 2 está associado ao aumento do risco de complicações micro e macrovasculares. Entre as complicações microvasculares destacam-se nefropatia, retinopatia e neuropatia; entre as macrovasculares as mais prevalentes são doença arterial coronariana (DAC), acidente vascular cerebral (AVC) e doença arterial obstrutiva periférica $(\mathrm{DAOP})^{(17)}$.

A anticoagulação natural exerce um papel importante no estabelecimento do equilíbrio hemostático e, para tal, depende da integridade vascular. Deficiências nesse sistema favorecem a formação de trombos.

A antitrombina (AT) é uma glicoproteína plasmática de 58KDa cuja ação anticoagulante caracteriza-se pela regulação da coagulação sangüínea promovendo a retirada de moléculas excedentes de trombina no processo e prevenindo a quebra do fibrinogênio em fibrina ${ }^{(6,13)}$. Para exercer sua função, a AT necessita de seu co-fator: a heparina. Substâncias semelhantes à heparina, como o sulfato de heparana e o sulfato de dermatana ${ }^{(13)}$, se encontram presentes no endotélio vascular. A Figura 1 mostra uma representação esquemática do mecanismo de ação da AT.

A proteína C (PC) é uma proteína de $62 \mathrm{KDa}$, dependente de vitamina K e um zimógeno circulante. Para exercer a sua função anticoagulante, a PC humana deve ser convertida à sua forma ativa: $\mathrm{PCa}^{(13)}$. A trombina tem como substrato preferencial o fibrinogênio, porém uma vez complexada à trombomodulina (TM), muda a sua conformação e passa a ter como substrato a PC, convertendo-a em PCa na presença de íons cálcio ${ }^{(11)}$. Para maior eficiência no processo de ativação, a PC antes se liga ao receptor de superfície (receptor endotelial de PC [EPCR]); e uma vez gerada, a PCa inativa os co-fatores Va e VIIIa ${ }^{(22)}$, como mostra a Figura 2.

Outra proteína plasmática envolvida nesse processo é a proteína S (PS). A PS é um co-fator cuja função é aumentar a ligação da $\mathrm{PCa}$ à membrana celular e acelerar a clivagem dos co-fatores pela enzima. PCa e PS formam um complexo 1:1 na presença de íons cálcio e fosfolípides de membrana. Quarenta por cento da PS encontra-se no plasma sob a forma livre e $60 \%$, ligada à C4bBP (proteína ligante da fração C $4 \mathrm{~b}$ do sistema do complemento). A PS é uma proteína dependente de vitamina $\mathrm{K}$ de $69 \mathrm{KDa}$, porém não é um zimógeno e sim um co-fator, como anteriormente mostrado ${ }^{(20)}$.

Ao contrário dos outros fatores da coagulação que atuam como enzimas (serinoproteases), os fatores VIII (FVIII) e V (FV) são co-fatores da cascata da coagulação, isto é, não possuem atividade catalítica. O fator VIlla é um co-fator na ativação do fator $\mathrm{X}$ em Xa pelo fator IXa. Na presença de íons cálcio e fosfolípides de membrana, a taxa de ativação do fator $X$ é aumentada mais de 10 mil vezes

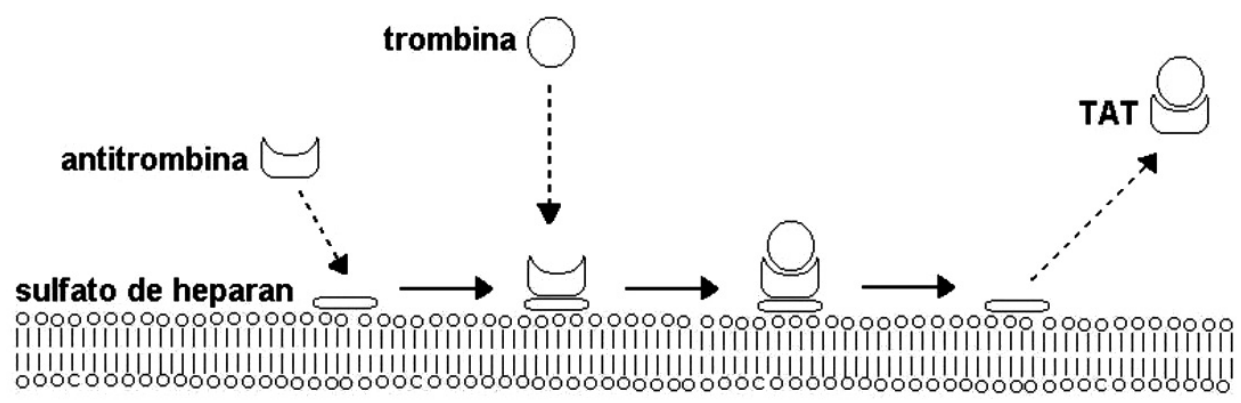

Figura 1 - Representação esquemática da inativação da trombina pela antitrombina, catalisada pelo sulfato de heparana, gerando o complexo trombina-antitrombina (TAT) 


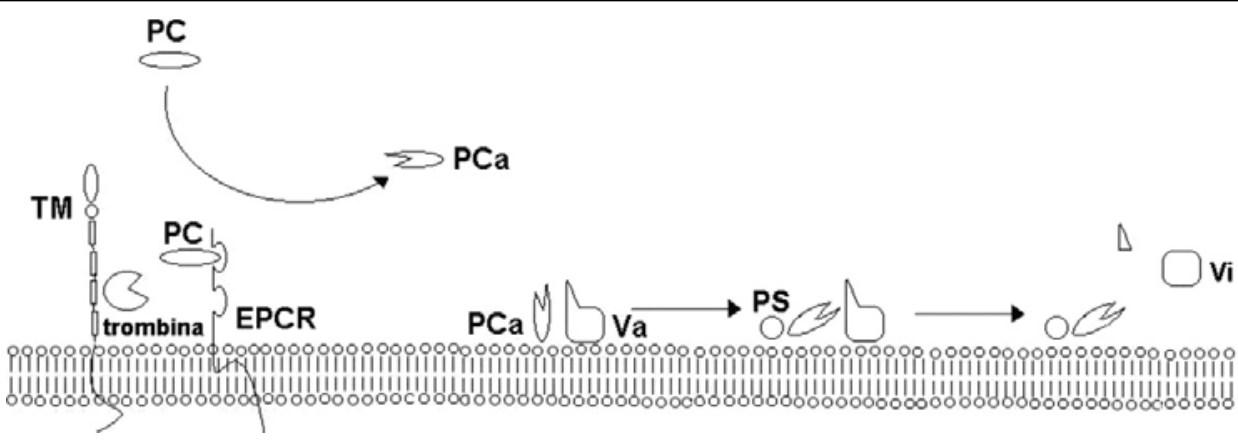

Figura 2 - Representação esquemática da ativação da PC em PCa e da inativação do fator Va pela PCa e o seu co-fator PS. PC: proteína C; PCa: proteína C ativada; TM: trombomodulina; EPCR: receptor endotelial para proteína C; Va: fator V ativado; PS: proteína S; Vi: fator V inativo

pelo fator VIIla. O fator Va é um co-fator na conversão de protrombina em trombina pelo fator Xa numa reação dependente de cálcio e fosfolípides. O complexo formado é denominado protrombinase e possui atividade catalítica, na clivagem da protrombina, 200 mil vezes maior que o Xa sozinho(6, 13, 20).

Os fatores Va e VIIla são inativados pela exposição prolongada à trombina ou pela PCa complexada ao seu co-fator PS e íons cálcio, um dos mecanismos da anticoagulação natural|(11).

\section{Objetivos}

O presente estudo teve como objetivo determinar os níveis plasmáticos de AT, PC, PS livre, e os níveis dos co-fatores V e VIII em pacientes com DM tipo 2, normoalbuminúricos, hipertensos e não-hipertensos para investigar o mecanismo da anticoagulação natural nesse grupo de risco aumentado para eventos trombóticos.

\section{Material e métodos}

Este estudo recebeu parecer favorável do Comitê de Ética em Pesquisa (CEP) da Santa Casa de Misericórdia de Belo Horizonte e do Comitê de Ética em Pesquisa (COEP) da UFMG.

Os 53 indivíduos participantes deste estudo compuseram quatro distintos grupos: 1) grupo-controle (controle), constituído de 16 indivíduos hígidos sob o ponto de vista clínico e laboratorial; 2) grupo com hipertensão (HAS), constituído de 12 indivíduos normoalbuminúricos com diagnóstico prévio de hipertensão; 3) grupo com DM tipo 2 (DM2), constituído de sete indivíduos normoalbuminúricos com diagnóstico prévio de DM tipo 2; 4) grupo com DM tipo 2 e hipertensão (DM2 + HAS), constituído de 18 indivíduos normoalbuminúricos com diagnóstico prévio de DM tipo 2 e hipertensão. Nenhum paciente apresentava, no momento da coleta, outras manifestações clínicas e laboratoriais.

Os indivíduos com diagnóstico clínico prévio de DM tipo 2, segundo os critérios da $\mathrm{OMS}^{(26)}$, ou que estivessem fazendo uso de medicamentos antidiabéticos foram classificados como DM tipo 2; os indivíduos com pressão sangüínea igual ou superior a $140 \mathrm{mmHg}$ para a pressão arterial sistólica ou $90 \mathrm{mmHg}$ para a pressão arterial diastólica, ou que estivessem fazendo uso de medicação anti-hipertensiva foram classificados como hipertensos ${ }^{(4)}$.

Foram excluídos do estudo indivíduos com doença renal crônica (concentração de creatinina sérica igual ou superior a $2 \mathrm{mg} / \mathrm{dl})^{(14)}$; indivíduos com bacteriúria e hematúria, com função anormal do fígado com alanina aminotransferase (ALT), aspartato aminotransferase (AST) ou fosfatase alcalina (FAL) com valores acima de duas vezes o limite superior de referência ${ }^{(10)}$; com microalbuminúria, ou seja, valores de albumina urinária superiores a $30 \mathrm{mg} / \mathrm{g}$ de creatinina ${ }^{(16,21)}$; com história clínica de doença cardíaca coronariana, AVC, tumor maligno, doença infecciosa aguda, ou que estavam em uso de medicamento com efeito sobre a função hemostática.

As amostras de sangue venoso foram obtidas entre $8 \mathrm{~h}$ e $10 \mathrm{~h}$ da manhã, após jejum de 12 a 14 horas. A coleta de sangue foi feita utilizando tubos do sistema Vacuette ${ }^{\circledR}$ (Geiner Bio-One), sendo 8ml em tubo sem anticoagulante (para as dosagens bioquímicas), $2 \mathrm{ml}$ em tubo com EDTA fluoretado (para a dosagem de glicose de jejum), $5 \mathrm{ml}$ em tubo com EDTA (para dosagem de glicoemoglobina) e 4,5ml em tubo com citrato de sódio 3,8\% (para as dosagens dos marcadores hemostáticos). Duas horas após o almoço foi colhida outra amostra de $2 \mathrm{ml}$ em tubo com EDTA fluoretado para a dosagem de glicose pós-prandial.

A amostra colhida em citrato foi centrifugada a $2.500 \mathrm{rpm}$ por 20 minutos e o plasma foi aliquotado, identificado e estocado a $-70^{\circ} \mathrm{C}$ até o momento da realização dos testes. 
As amostras de urina foram coletadas em frascos apropriados, após higienização prévia. Foi coletado o jato médio da primeira urina da manhã ou da segunda, após três horas de retenção. A urina foi centrifugada a 1.500rpm por cinco minutos e processada em no máximo duas horas. Parte da urina centrifugada foi aliquotada, identificada e estocada a $-70^{\circ} \mathrm{C}$ para posterior determinação da microalbuminúria.

Glicemia, colesterol total (CT), HDLc, triglicérides (TCL), ALT, AST, uréia e creatinina foram determinados usando kits da Bioclin ${ }^{\circledR}$ e equipamento BTR-811 (Biotron ${ }^{\circledR}$ ). Microalbuminúria foi determinada usando kit Biotécnica ${ }^{\circledR}$ e equipamento $\mathrm{BIO} 2000$ (BioPlus ${ }^{\circledR}$ ). Eletrólitos sódio e potássio foram quantificados por fotometria de chama no equipamento Cornig $400^{\circledR}$.

A determinação da $\mathrm{PC}$ foi realizada através do uso do conjunto diagnóstico TEClot Protein $\mathrm{C}^{\circledR}\left(\mathrm{Teco}^{\circledR} \mathrm{GmbH}\right.$ - Alemanha), utilizando-se o aparelho ST4BIO (Stago ${ }^{\circledR}$ ) pelo método coagulométrico. A PS livre foi determinada pelo método de ELISA através do uso do conjunto diagnóstico Imuclone ${ }^{\circledast}$ Free Protein S (American Diagnostica ${ }^{\circledast}$ Inc. - Estados Unidos). A AT foi determinada pelo método cromogênico cinético através do uso do conjunto diagnóstico Spectrolyse ${ }^{\circledast}$ Antithrombin III (Biopoo ${ }^{\circledR}$ - Estados Unidos) e utilizando-se o aparelho Packs-4 (Helena Laboratories USA ${ }^{\circledR}$ ). Os fatores $\mathrm{V}$ e VIII foram determinados pelo método coagulométrico através do uso dos conjuntos diagnósticos

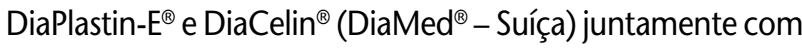
o Plasmas Deficientes em fator V e VIII (DiaMed ${ }^{\circledR}$ - Suíça) e utilizando-se o aparelho ST4BIO $\left(\right.$ Stago $\left.^{\circledR}\right)$.

\section{Análise estatística}

Os resultados dos grupos estão expressos pelos valores das médias e dos desvios-padrão. Foi realizado o teste de análise de variância multipareada para comparação dos grupos, utilizando-se o programa Sigma Stat ${ }^{\circledR}$ versão 1.0. Valor de $p$ menor que 0,05 foi considerado estatisticamente significante. $O$ teste de análise de variância (Anova) foi utilizado para os parâmetros que apresentaram distribuição normal e homocedasticidade. $O$ teste de análise de variância Kruskal-Wallis foi utilizado para os parâmetros que não apresentaram distribuição normal e/ou homocedasticidade.

\section{Resultados}

A Tabela 1 apresenta as características clínicas e metabólicas dos grupos controle, HAS, DM2 e DM2 + HAS. Os resultados dos parâmetros hemostáticos obtidos neste trabalho encontram-se na Tabela 2. Os dados estão apresentados com os valores das médias e dos desvios-padrão para os grupos.

Em relação aos níveis de AT, o grupo HAS apresentou valor de média mais elevado em relação às médias dos grupos controle e DM2, porém não foi observada diferença significativa quando comparado com o grupo DM2 + HAS (Figura 3). Até o momento não se sabe como a HAS poderia interferir nos níveis de AT, tampouco se esse aumento poderia ser um mecanismo adaptativo para prevenção de eventos trombóticos.

A análise da Figura 4 permite observar que a média dos níveis plasmáticos de $\mathrm{PC}$ foi significativamente maior no grupo DM2 + HAS em relação à média do grupo controle. Não houve diferença significativa entre os demais grupos, porém pode-se observar uma tendência de aumento no grupo DM2.

Para os níveis plasmáticos de PS livre não foram encontradas diferenças significativas entre as médias dos grupos estudados (Figura 5). Esse fato sugere que nem a hipertensão nem o DM tipo 2 alteram os níveis de PS livre, sendo esta proteína menos susceptível ao fator que provocou aumento dos níveis de PC.

$\mathrm{Na}$ Figura 6 pode-se observar que a média dos níveis plasmáticos de FV do grupo DM2 + HAS foi significativamente maior que as médias dos grupos controle e DM2.

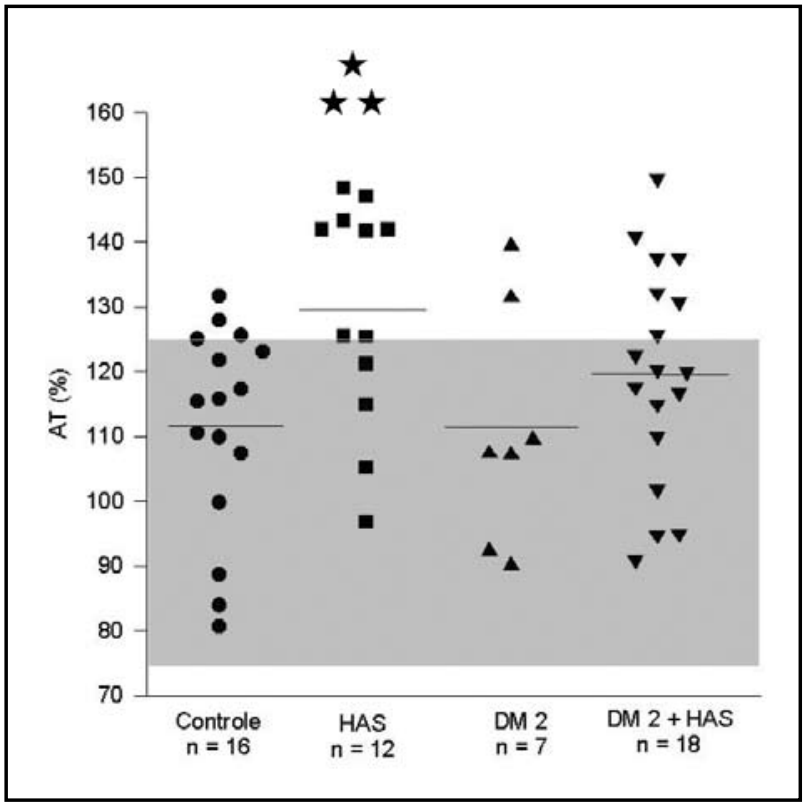

Figura 3 - Distribuição dos valores de antitrombina (AT). Valores expressos em porcentagem para os grupos controle, HAS, DM2 e DM2 + HAS. A área sombreada corresponde à faixa de normalidade (75\% a 125\%), as linhas horizontais representam as médias dos grupos, $\star$ indica diferença significativa em relação ao grupo controle $(p=0,008)$ $e \star \star$ indica diferença significativa em relação ao grupo DM2 $(p=0,04)$ 
Tabela 1 Características clínicas e metabólicas dos grupos estudados

\begin{tabular}{lccccc}
\hline & Controle & HAS & DM2 & DM2 + HAS & $p$ \\
$n$ (m/h) & $16(9 / 7)$ & $12(8 / 4)$ & $7(3 / 4)$ & $18(16 / 2)$ & \\
Idade (anos) & $52,3 \pm 5,4$ & $54,8 \pm 5,2$ & $52,1 \pm 8,3$ & $57,2 \pm 6$ & \\
IMC (kg/m $)$ & $25,1 \pm 3,9$ & $28,4 \pm 3,6$ & $28,1 \pm 3,5$ & $28,6 \pm 4,1$ & \\
GLI (mg/dl) & $91,7 \pm 9,3$ & $91 \pm 9,9$ & $154,6 \pm 55,2^{\mathrm{a}, \mathrm{b}}$ & $167,6 \pm 57,3^{\mathrm{a}, \mathrm{b}}$ & $p^{\mathrm{a}}<0,0001$ \\
& & & & $\mathrm{p}^{\mathrm{b}}<0,0001$ \\
GHB (\%) & $6,4 \pm 1,5$ & $7,2 \pm 2,1$ & $8,6 \pm 3,2$ & $8,9 \pm 2,9^{\mathrm{a}}$ & $p^{\mathrm{a}}=0,02$ \\
GLI PP (mg/dl) & $102,8 \pm 16,6$ & $103,3 \pm 19,8$ & $184,7 \pm 122,1$ & $151,5 \pm 69,2^{\mathrm{a}, \mathrm{b}}$ & $p^{\mathrm{a}}=0,01$ \\
& & & & $p^{\mathrm{b}}=0,04$ \\
CT (mg/dl) & $180,4 \pm 27,9$ & $198,4 \pm 44,5$ & $201,7 \pm 44,2$ & $190,6 \pm 44,5$ & $\mathrm{~ns}$ \\
HDLc (mg/dl) & $47,5 \pm 10,1$ & $40 \pm 13,2$ & $41 \pm 7$ & $39,6 \pm 12^{\mathrm{a}}$ & $p^{\mathrm{a}}=0,046$ \\
LDLc (mg/dl) & $116,3 \pm 29,8$ & $129,9 \pm 39,7$ & $137,4 \pm 46$ & $117,5 \pm 37$ & $\mathrm{~ns}$ \\
TGL (mg/dl) & $82,9 \pm 45,2$ & $142,5 \pm 69,2^{\mathrm{a} 1}$ & $116,7 \pm 53,1$ & $168 \pm 75^{\mathrm{a} 2}$ & $p^{\mathrm{a} 1}=0,009$ \\
& & & & $p^{\mathrm{a} 2}=0,0004$ \\
ALT (U/l) & $22,5 \pm 9,4$ & $18,8 \pm 10,6$ & $22,3 \pm 8,8$ & $23,6 \pm 13,3$ & $\mathrm{~ns}$ \\
AST (U/l) & $27,8 \pm 8,3$ & $21,4 \pm 4,8$ & $20,3 \pm 6,5$ & $23,5 \pm 13,4$ & $\mathrm{~ns}$ \\
FAL (U/l) & $29,5 \pm 9,2$ & $38,5 \pm 10^{\mathrm{a} 1}$ & $39,7 \pm 15,7$ & $40,9 \pm 11,4$ a2 & $p^{\mathrm{a} 1}=0,02$ \\
& & & & $p^{\mathrm{a} 2}=0,003$ \\
Na+ (mEq/l) & $139,6 \pm 6,2$ & $142,8 \pm 6,5$ & $137,7 \pm 6,6$ & $138,6 \pm 7,9$ & $\mathrm{~ns}$ \\
$\mathrm{~K}^{+}(\mathrm{mEq} / \mathrm{l})$ & $3,8 \pm 0,5$ & $4,1 \pm 0,4$ & $3,7 \pm 0,5$ & $4,5 \pm 0,7^{\mathrm{a}, \mathrm{c}}$ & $p^{\mathrm{a}}=0,02$ \\
& & & & $p^{\mathrm{c}}=0,03$ \\
URE (mg/dl) & $22,5 \pm 5,5$ & $22 \pm 7,6$ & $23,9 \pm 9,5$ & $26,1 \pm 10,3$ & $\mathrm{~ns}$ \\
CRE (mg/dl) & $1,1 \pm 0,3$ & $1,1 \pm 0,2$ & $0,9 \pm 0,3 \mathrm{~b} 1$ & $0,8 \pm 0,3^{\mathrm{a}, \mathrm{b} 2}$ & $p^{\mathrm{a}}=0,03$ \\
Alb U/Cr (mg/g) & $5,5 \pm 2,9$ & $9,1 \pm 7,9$ & $5,1 \pm 2,5$ & $8,8 \pm 6,6$ & $p^{\mathrm{b} 1}=0,03$ \\
\hline
\end{tabular}

Caracterização quanto a n (tamanho), $m$ (número de mulheres) e h (número de homens), idade e IMC (índice de massa corporal), sendo os valores dos dois últimos parâmetros expressos como média e desvio-padrão. Os dados bioquímicos estão representados pelas médias e pelos desvios-padrão dos valores de GLI (glicemia de jejum), GHB (glicoemoglobina), GLI PP (glicemia pós-prandial), CT (colesterol total), HDLC (colesterol presente na lipoproteína de alta densidade), LDLc (colesterol presente na lipoproteína de baixa densidade), TGL (triglicérides), ALT (alanina aminotransferase), AST (aspartato aminotransferase), FAL (fosfatase alcalina), Na (sódio), $K^{+}$(potássio), URE (uréia), CRE (creatinina) e Alb $\mathrm{U} / \mathrm{Cr}$ (relação albumina/creatinina urinária) para os grupos controle, HAS, DM2 e DM2 + HAS. As diferenças significativas entre os grupos estão representadas pelas letras: a (vs. controle), b (vs. HAS) ec (vs. DM2).

Tabela 2 Parâmetros hemostáticos

\begin{tabular}{lcccc}
\hline & Controle & HAS & DM2 & DM2 + HAS \\
PC (\%) & $124,9 \pm 19,6$ & $133,3 \pm 21,7$ & $145 \pm 33,7$ & $143,2 \pm 21,6^{\mathrm{a}}$ \\
PS livre (\%) & $87,8 \pm 19,5$ & $88,8 \pm 9,7$ & $85,1 \pm 8,2$ & $90,9 \pm 12,8$ \\
AT (\%) & $111,5 \pm 15,8$ & $129,5 \pm 17,3^{\mathrm{a}, \mathrm{c}}$ & $111,1 \pm 18,5$ & $120 \pm 16,8$ \\
Fator V (\%) & $112,2 \pm 27,1$ & $122,2 \pm 17$ & $105,3 \pm 18,9$ & $133 \pm 28,4^{\mathrm{a}, \mathrm{c}}$ \\
Fator VIII (\%) & $108,3 \pm 34,1$ & $117,7 \pm 34$ & $112,8 \pm 46,4$ & $112 \pm 45,8$ \\
\hline
\end{tabular}

Os dados estão representados pelas médias e pelos desvios-padrão dos valores plasmáticos de AT (antitrombina), PC (proteína C), PS livre (proteína S livre), FV (fator V) e FVIII (fator VIII) para os grupos controle, HAS, DM2 e DM2 + HAS. As diferenças significativas entre os grupos estão representadas pelas letras: a (vs. controle), b (vs. HAS) e c (vs. DM2). 


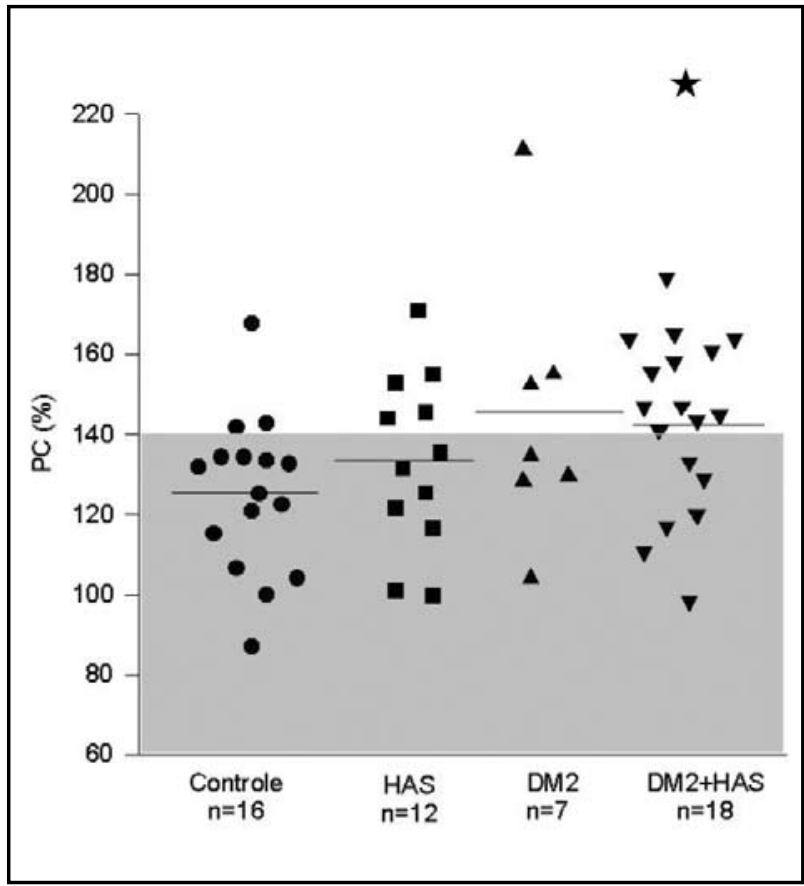

Figura 4 - Distribuição dos valores de proteína $C(P C)$. Valores expressos em porcentagem para os grupos controle, HAS, DM2 e DM2 + HAS. A área sombreada corresponde à faixa de normalidade (60\% a 140\%), as linhas horizontais representam as médias dos grupos e o símbolo « indica diferença significativa em relação ao grupo controle $(p=0,015)$



Figura 5 - Distribuição dos valores de proteína S livre (PS livre). Valores expressos em porcentagem para os grupos controle, HAS, DM2 e DM2 + HAS. A área sombreada corresponde à faixa de normalidade (60\% a 150\%) e as linhas horizontais representam as médias dos grupos. Não houve diferença significativa entre os grupos

Para os demais grupos não houve diferença significativa entre as médias. Embora a média do grupo DM2 + HAS tenha apresentado valor acima do de referência, em todos os grupos foram encontrados resultados aumentados.

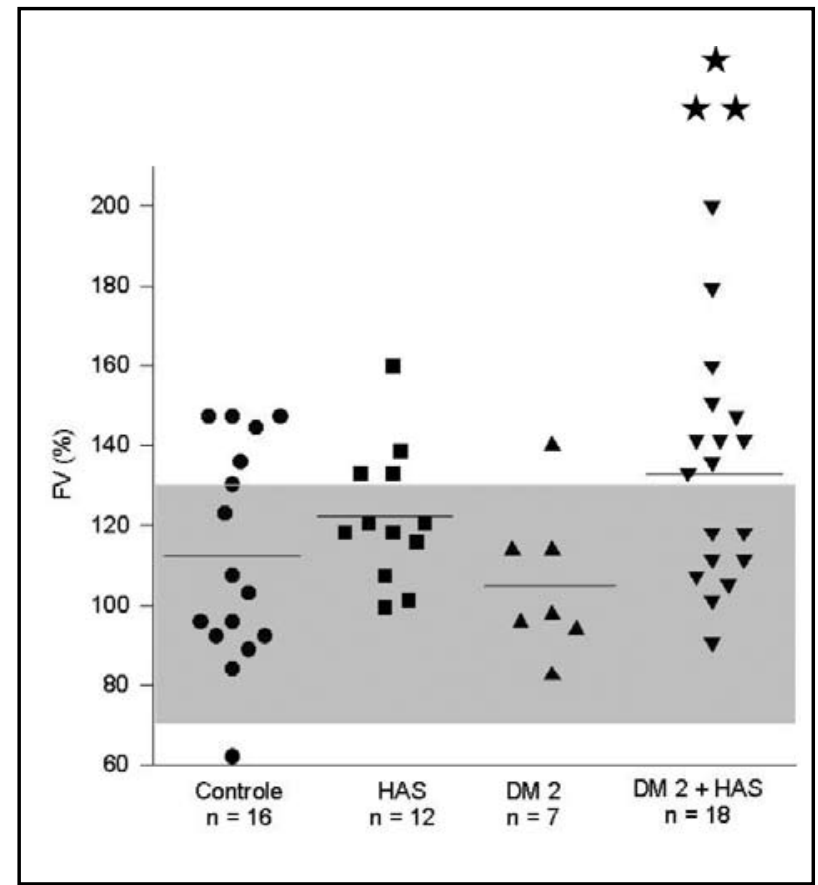

Figura 6 - Distribuição dos valores de fator $V(F V)$. Valores expressos em porcentagem para os grupos controle, HAS, DM2 e DM2 + HAS. A área sombreada corresponde à faixa de normalidade (70\% a 130\%), as linhas horizontais representam as médias dos grupos, $\star$ indica diferença significativa em relação ao grupo controle $(p=0,04) e \star \star$ indica diferença significativa em relação ao grupo DM2 $(p=0,03)$.

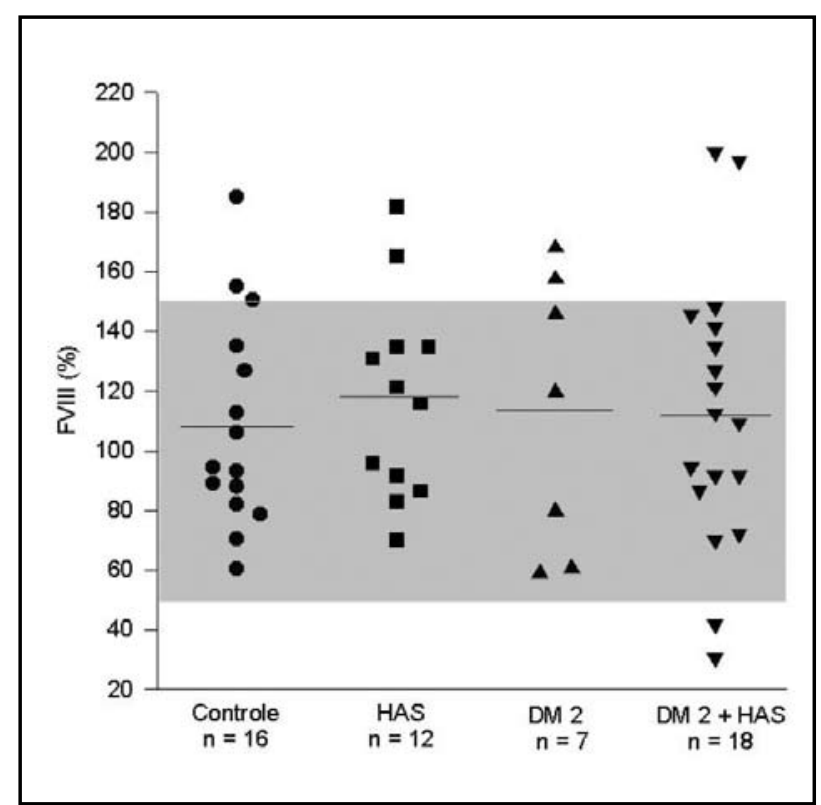

Figura 7 - Distribuição dos valores de fator VIII (FVIIII). Valores expressos em porcentagem para os grupos controle, HAS, DM2 e DM2 + HAS. A área sombreada corresponde à faixa de normalidade (50\% a 150\%) e as linhas horizontais representam as médias dos grupos. Não houve diferença significativa entre os grupos

A Figura 7 apresenta os dados obtidos na determinação da concentração plasmática de FVIII, expressos em porcentagem. Não foi encontrada diferença significativa entre os grupos controle, HAS, DM2 e DM2 + HAS. Todos 
os grupos apresentam alguns resultados acima do valor de referência.

\section{Discussão}

Como o sistema hemostático é altamente dependente da integridade vascular, é de grande importância estimar o grau de comprometimento microvascular que os indivíduos diabéticos e hipertensos apresentam. Entre as principais manifestações da microangiopatia podem-se destacar retinopatia, neuropatia e nefropatia. A retinopatia e a neuropatia são avaliadas por exames clínicos e caracterizadas qualitativamente. Já a nefropatia é avaliada principalmente pela excreção urinária de albumina, que é um parâmetro quantitativo. Por esse motivo, no presente estudo foi realizada a determinação da relação Alb $\mathrm{U} / \mathrm{Cr}$ para avaliar o comprometimento microvascular. Todos os indivíduos participantes deste estudo eram normoalbuminúricos, porém essa informação isolada não descarta a hipótese de possível alteração endotelial e comprometimento do sistema hemostático.

Sabe-se que a hiperglicemia promove uma redução da atividade biológica da AT em indivíduos diabéticos, porém que a concentração do antígeno pode ser normal ou até um pouco aumentada ${ }^{(25)}$. Modificações estruturais ocasionadas pela glicação não-enzimática foram sugeridas como o fator responsável pela disfunção ${ }^{(3,25)}$. Esse motivo pode em parte justificar a menor atividade de AT no grupo DM2 + HAS em relação ao grupo HAS. O grupo DM2 + HAS apresentou um caráter intermediário entre os grupos DM2 e HAS.

Vários estudos já encontraram níveis reduzidos, normais e aumentados de AT em indivíduos diabéticos, não havendo um senso comum. Tal fato possivelmente está associado à presença de co-variáveis que alteram e confundem o quadro e comumente são observadas em indivíduos diabéticos (estágio de comprometimento microvascular, presença de co-morbidades como hipertensão, hipotireoidismo, processos inflamatórios, entre outros fatores) ${ }^{(2)}$.

$\mathrm{O}$ aumento da atividade, bem como da concentração antigênica da proteína $C(P C)$, em pacientes com DM tipo 2 com normo e microalbuminúria já foi descrito na literatura, sendo que os com normoalbuminúria apresentaram média mais elevada ${ }^{(1,12)}$. Sabe-se que a PC usualmente está aumentada em pacientes com diabetes e que ela não é uma proteína de fase aguda ${ }^{(24)}$.

O mecanismo pelo qual ocorre o aumento de PC em indivíduos com DM tipo 2 ainda não foi consolidado pela literatura. Esse aumento poderia estar relacionado a alguma alteração característica da doença, como a hiperglicemia e a glicação de proteínas.

Indivíduos idosos também apresentam valores de PC mais elevados que jovens. Na doença cardíaca isquêmica, durante a gravidez, no período pós-menopausa, em mulheres em uso de terapia de reposição hormonal e em uso de anticoncepcionais orais também já foram relatados aumentos dos níveis de PC. Porém, não há nenhuma significância clínica conhecida para esse aumento, exceto que ele pode dificultar a detecção da deficiência funcional de PC(15).

O aumento dos níveis de PC detectado laboratorialmente deve ser interpretado com cautela. $\mathrm{O}$ aumento do antígeno e/ou o aumento da atividade in vitro da PC não necessariamente reflete uma maior atividade da $\mathrm{PC}$ in vivo. A PC pode não estar sendo ativada, uma vez que esse processo é dependente da interação com o endotélio íntegro, a trombomodulina, o EPCR e a trombina.

A determinação de PS livre atualmente é a melhor metodologia para dosagem de PS, pois avalia a forma funcional da proteína no sistema da anticoagulação(23).

Como os níveis de PS livre não variaram entre os grupos, pode-se admitir que processos inflamatórios subclínicos não estavam presentes nesses pacientes, pois se sabe que em condições normais, $60 \%$ de PS circula complexada à C4bBP e 40\%, na forma livre (fração com atividade anticoagulante). Como C4bBP é uma proteína de fase aguda, aumentando em processos inflamatórios, seria esperada uma redução dos níveis de PS livre pelo deslocamento do equilíbrio, o que justificaria em parte o estado de hipercoagulabilidade nas doenças inflamatórias ${ }^{(7)}$.

É descrito na literatura que no DM tipo 2 há um expressivo aumento da ativação das plaquetas $(5,9,18)$. Sabendo que o FV é armazenado nos grânulos das plaquetas, é de se esperar sua liberação quando ocorre ativação plaquetária. Entretanto, o aumento de FV foi expressivo no grupo DM2 + HAS e não no grupo DM2 e, dessa forma, acredita-se que a associação com HAS deva contribuir para o aumento de FV.

Uma vez ativada a cascata da coagulação, níveis aumentados de FVa podem favorecer ainda mais a formação de fibrina. O FVa é inativado pela PCa, entretanto mesmo estando o sistema da anticoagulação natural completamente funcionante, ele pode não conseguir inativar todo o FVa (presente em excesso), permanecendo, dessa forma, quantidades suficientes para a formação do complexo protrombinase que favorece a formação de fibrina. 
Vários estudos já observaram aumento da atividade do FVIII em diabéticos e posterior normalização com o tratamento da doença. Nos pacientes diabéticos com microalbuminúria os níveis de FVIII e FvW normalmente encontram-se aumentados ${ }^{(2)}$.

Porém, torna-se oportuno enfatizar que os pacientes do presente estudo eram diabéticos normoalbuminúricos, o que pode justificar em parte a não-observação de diferença significativa entre pacientes e controles.

Outro ponto que reforça esse achado é a concordância com os níveis de PS livre, uma vez que, assim como a C4bBP, o FVIII também é uma proteína de fase aguda. Da mesma forma que não foi observada redução de PS livre, também não houve aumento de FVIII.

\section{Conclusões}

No presente estudo foi possível observar que indivíduos diabéticos, hipertensos e diabéticos hipertensos não apresentaram deficiência dos níveis plasmáticos dos anticoa- gulantes naturais, fato que poderia estar associado à alta freqüência de eventos trombóticos nessa população.

Deficiências quantitativas e qualitativas de PC, PS e AT são fatores de risco bem estabelecidos para o tromboembolismo venoso, porém níveis aumentados não proporcionam redução do risco de desenvolvimento de trombose e até o momento não apresentam qualquer significância clínica ${ }^{(15,23)}$.

Admite-se, então, que nos indivíduos com DM tipo 2, hipertensão e normoalbuminúria, um processo inflamatório importante não deve fazer parte da patogênese dessa doença, ou seja, não altera a anticoagulação natural em relação aos níveis de PS livre.

O aumento de FV nos indivíduos diabéticos hipertensos pode em parte favorecer a formação de trombos; já os níveis normais de FVIII apontam para uma eficiência do sistema hemostático.

Para melhor elucidar essa questão, estudos mais amplos são necessários para o entendimento das alterações que ocorrem nos indivíduos diabéticos.

\section{Referências}

I. ASO, Y. et al. Relationship between soluble thrombomodulin in plasma and coagulation or fibrinolysis in type 2 diabetes. Clin Chim Acta, v. 30 I, n. I-2, p. I 35-45, 2000

2. CARR, M. E. Diabetes mellitus: a hypercoagulable state.J Diabetes Complications, v. I5, n. I, p. 44-54, 2001

3. CERIELLO, A. et al. Induced hyperglycemia alters antithrombin III activity but not its plasma concentration in healthy normal subjects. Diabetes, v. 36, n. 3, p. 320-3, 1987.

4. CHOBANIAN,A.V. et al. National High Blood Pressure Education Program Coordinating Committee. The seventh report of the Joint National Committee on prevention, detection, evaluation, and treatment of high blood pressure:The JNC 7 report.JAMA, v. 289, n. 19, p. 2560-72, 2003.

5. COMBES, $\vee$. et al. Increased levels and procoagulant activity of microparticles in diabetes patients. In: XVIII INTERNATIONAL SOCIETY ON THROMBOSIS AND HAEMOSTASIS (ISTH), 200I, Paris. Resumo. CD-ROM

6. DAHLBÄCK, B. Blood coagulation. Lancet, v. 355, n. 92। 5, p. 1627-32, 2000

7. D'ANGELO, $A$. et al. Protein $S$ and protein $C$ anticoagulant activity in acute and chronic cardiac ischemic syndromes. Relationship to inflammation, complement activation and in vivo thrombin activity. Thromb Res, v. 75, n. 2 p. $133-42$, 1994. Apud: BOULLANGER, N. et al. Coagulation activation in patients with an inflammatory syndrome: is there a link with acquired protein S deficiency? Blood Coagul Fribrinolysis, v. 9, n. 2, p. |67-7|, 1998

8. DeFRONZO, R. A. Insulin resistance: a multifaceted syndrome responsible for NIDDM, obesity, hypertension, dyslipidaemia and atherosclerosis. Neth J Med, v. 50, n. 5, p. 191-7, 1997.

9. DIAMANT, M. et al. Cell-derived microparticules in patients with uncomplicated type 2 diabetes mellitus are procoagulant. In: XVIII INTERNATIONAL SOCIETY ONTHROMBOSIS AND HAEMOSTASIS (ISTH), 200 I, Paris. Resumo. CDROM.

I0. DUFOUR, R. et al. Diagnosis and monitoring of hepatic injury. II. Recommendations for use of laboratory tests in screening, diagnosis, and monitoring. Clin Chem, v. 46, n. 12, p. 205068, 2000 .

I I. ESMON, C.T. Regulation of blood coagulation. Biochim Biophys Acta, v. 1477, n. 2, p. 49-360, 2000.

12. GABAZZA, E. C. et al. Protein C activation in NIDDM patients. Diabetologia, v. 39, n. 12, p. |455-61, 1996.

1 3. HANDIN, R. I.; LUX, S. E.; STOSSEL,T.P.(Ed.). Blood: principles of hematology. Philadelphia: J.B. Lippincott, 1995. 2305p.

14. HIRANO, T. et al.Vascular endothelial markers, von Willebrand factor and thrombomodulin index, are specifically elevated in type 2 diabetic patients with nephropathy: comparison of primary renal disease. Clin Chim Acta, v. 299, n. 1-2, p. $65-75,2000$

15. KOTTKE-MARCHANT, K.; COMP, P. Laboratory issues in 
diagnosing abnormalities of protein $\mathrm{C}$, thrombomodulin, and endothelial cell protein C receptor. Arch Pathol Lab Med, v. 126, n. II, p. 1337-48, 2002.

16. MOLITCH, M. E. et al. Diabetic nephropathy. Diabetes Care, v. 26, p. S94-98, 2003. Suplemento I.

17. NAZIMEK-SIEWNIAK, B. et al. Risk of macrovascular and microvascular complications in type 2 diabetes results of longitudinal study design. J Diabetes Complications, v. 16, n. 4, p. 27I-6, 2002.

18. OUVIÑA, S. M. et al. Endothelial dysfunction, nitric oxide and platelet activation in hypertensive and diabetic type II patients. Thromb Res, v. 102, n. 2, p. 107- 14, 2001.

19. SBD (SOCIEDADE BRASILEIRA DE DIABETES). Consenso Brasileiro sobre Diabetes. Diagnóstico e classificação do diabetes mellitus e tratamento do diabetes mellitus tipo 2, 2000. Disponível em: <http://www.diabetes.org.br/ consenso/index.html>. Acesso em: 27 set. 2002.

20. SCAZZIOTA, A.; ALTMAN, R. El mecanismo de la hemostasia normal. Revista Iberoamericana de Trombosis y Hemostasia, v. I, p. 9-29, 1996.

2।. STEHOUWER, C. D. A.; GALL, M.; TWISK, J. W. R. et al. Increased urinary albumin excretion, endothelial dysfunction, and chronic low-grade inflammation in type 2 diabetes: prospective, interrelated, and independently associated with risk of death. Diabetes, v. 5I, n. 4, p. I I 57-65, 2002.
22.TAYLOR Jr, F. B.; PEER, G.T.; LOCKHART, M. S. et al. Endothelial cell protein $C$ receptor plays an important role in protein C activation in vivo. Blood, v. 97, n. 6, p. 1685-8, 2001.

23. TRIPODI, A.; MANNUCCI, P. M. Laboratory investigation of thrombophilia. Clin Chem, v. 47, n. 9, p. 1597-606, 2001.

24.VIGANO, S.; MANNUCCI, P. M.; D'ANGELO, A. et al. Protein C is not an acute phase reactant and is often high in ischemic heart disease and diabetes. Thromb Haemost, v. 52, n. 3, p. 263-6, 1984.

25. VILLANUEVA, G. B.; ALLEN, N. Demonstration of altered antithrombin III activity due to nonenzymatic glycosylation at glucose concentration expected to be encountered in severely diabetic patients. Diabetes, v. 37, n. 8, p. I 103-7, 1988.

26.WHO (WORLD HEALTH ORGANIZATION). Department of Noncommunicable Disease Surveillance. Definition, diagnosis and classification of diabetes mellitus and its complications. Geneva, 1999. 59p.

27. WHO (WORLD HEALTH ORGANIZATION). Diabetes mellitus. Fact sheets, n. 138, 2002. Disponível em: <http:// www.who.int/inf-fs/en/fact l 38.html>. Abril 2002. Acesso em: 27 set. 2002.

28. ZIMMET, P.; ALBERT, K. G. M. M.; SHAW, J. Global and societal implications of the diabetes epidemic. Nature, v. 4I4, p. 782-7, 200 I. 